\title{
Lifesaving Intraosseous Access in a Patient with a Massive Obstetric Hemorrhage
}

\author{
Joey de Vogel, M.D., 1 Roger Heydanus, M.D., Ph.D., 1 \\ Annemarie G.M. Mulders, M.D., Ph.D., Dina J.C. Smalbraak, M.D., 1 \\ Dimitri N.M. Papatsonis, M.D., Ph.D., 1 and Bastiaan M. Gerritse, M.D., Ph.D. ${ }^{2}$
}

A 42-year-old, gravida 1, para 0 woman was induced at a gestational age of 41 weeks because of post-term dates. The fourth stage of delivery was complicated by a massive hemorrhage. The uncontrollable persisting amount of blood loss led to hypovolemic shock and cardiopulmonary arrest. Lifesaving extra access was gained through an intraosseous needle in the proximal tibia. We therefore advocate including the use of an intraosseous needle as an additional route for intravascular volume replacement in case of peripartum hemorrhage.

KEYWORDS: Intraosseous infusion, massive obstetric hemorrhage, peripartum, hypovolemic shock

Postpartum hemorrhage (PPH) remains a leading cause of maternal morbidity and mortality despite modern improvements in obstetric practice and transfusion services. PPH is defined as a blood loss more than $500 \mathrm{~mL}$ within 24 hours after birth, and severe $\mathrm{PPH}$ as blood loss greater than $1000 \mathrm{~mL}$ within 24 hours. ${ }^{1}$ Vaginal deliveries are complicated by severe $\mathrm{PPH}$ in 4 to $5 \%$ of cases. ${ }^{2}$ Blood loss in cesarean section is 50 to $100 \%$ more than blood loss during vaginal delivery. ${ }^{3}$ In some developed countries, the incidence of $\mathrm{PPH}$ is increasing. ${ }^{4}$ In the United Kingdom, obstetric hemorrhage is the sixth most common cause of maternal death in the triennium 2006 to $2008 .{ }^{5}$ Obstetric hemorrhage is often associated with other adverse postoperative complications such as sepsis and acute renal failure requiring admission to the intensive care unit. ${ }^{6}$ In our unit, we therefore try to minimize blood loss during delivery by an active management of the third stage of labor. ${ }^{7}$ However, when a severe PPH occurs, optimizing all other treatment options is essential. We present a case of severe massive obstetric hemorrhage, which was successfully managed by multidisciplinary team approach, quick decision making, and the use of intraosseous access needle for infusion in the proximal tibia.

\section{CASE REPORT}

A healthy 42-year-old, gravida 1 , para 0 woman was admitted at 41 weeks' gestation for an elective induction of labor because of post-term dates; she was induced with vaginal prostaglandin E2 gel (Prostin ${ }^{\circledR}$; Pfizer BV, Capella aan den Ussel, The Netherlands) for cervical ripening because of an unfavorable cervix. Pregnancy had been uneventful. Her past medical history was also uneventful. After 9 hours at $4 \mathrm{~cm}$ of dilatation, the membranes were artificially ruptured and augmentation of labor was started with intravenous oxytocin (Syntocinon ${ }^{\mathbb{R}}$; Novartis Pharma BV, Arnhem, The Netherlands). The first stage of labor was uneventful, and in 10 hours complete dilatation was achieved. After a
Departments of ${ }^{1}$ Obstetrics and Gynecology, and ${ }^{2}$ Anaesthesiology, Amphia Hospital Breda, Breda, The Netherlands.

Address for correspondence and reprint requests: Dimitri N.M. Papatsonis, M.D., Ph.D., Department of Obstetrics and Gynecology, Amphia Hospital Breda, Langendijk 75, 4819 EV Breda, The Netherlands (e-mail: Hoog.pap@wxs.nl).

Am J Perinatol Rep 2011;1:119-122. Copyright (C) 2011 by
Thieme Medical Publishers, Inc., 333 Seventh Avenue, New York, NY 10001, USA. Tel: +1 (212) 584-4662.

Received: July 3, 2011. Accepted after revision: August 15, 2011. Published online: November 11, 2011.

DOI: http://dx.doi.org/10.1055/s-0031-1293514.

ISSN 2157-6998. 
second stage of 62 minutes and a left mediolateral episiotomy, a healthy male infant of $3980 \mathrm{~g}$ was delivered spontaneously. According to our protocol on active management of the third stage of labor, a bolus of intravenous oxytocin (5 IU) was given, ${ }^{7}$ and the placenta was delivered by controlled cord traction after 6 minutes. The episiotomy was sutured uneventfully. The fourth stage of delivery was complicated by a massive blood loss 45 minutes after delivery. Despite the use of several uterotonic agents such as extra intravenous oxytocin (5 IU), intravenous methyl-ergometrine (Methergin ${ }^{\circledR}$; Novartis Pharma BV, Arnhem, The Netherlands) 0.2 $\mathrm{mg}$, misoprostol (Cytotec ${ }^{\mathbb{R}}$; Pfizer BV, Capella aan den Ussel, The Netherlands) $800 \mu \mathrm{g}$ rectally, and intravenous sulprostone (Nalador ${ }^{\mathbb{R}}$; Schering Nederland BV, Weesp, The Netherlands) $500 \mu \mathrm{g}$ in 15 minutes, there was persisting massive vaginal blood loss of 2000 $\mathrm{mL}$ in a 30 -minute period in combination with uterine atony. Vital parameters demonstrated blood pressure of $100 / 70 \mathrm{~mm} \mathrm{Hg}$, pulse rate of 112 beats per min, and respiration rate of 20 per minute. Supplementary highflow oxygen was administered through a non-rebreathing bag valve mask. A second large-bore 14-gauge intravenous line was inserted, and fluid replacement was started. In consultation with the anesthesiologist, it was decided to transport the patient to the operating theater for further stabilization and examination under general anesthesia. Blood transfusion was started. On examination under anesthesia, there were no signs of vulvovaginal or external cervical laceration contributing to the massive blood loss. There was uterine atony only temporarily responding to uterotonic agents and uterine massage and compression. The predominant origin of the bleeding seemed to occur from a region internally left inside the cervical canal. Vaginally, a tooth forceps was placed and left inside the internal cervical os, resulting in temporal reduction of the uterine bleeding. Because of the unstable cardiovascular situation despite these measures, laparotomy was performed by a midline skin incision. After massage and bimanual compression of the uterus, a modified B-Lynch brace-type suture was inserted and tied with ligation of the uterine arteries. Despite this, massive blood loss persisted. Because of the unstable situation with vital parameters progressing toward hypovolemic shock, selective embolization of the uterine arteries by the radiologist was not an option. Despite aggressive volume replacement, it was impossible to compensate for the continuing amount of blood loss through the two large-bore peripheral intravenous lines. Hence, the only possibility to gain rapid access to the circulation was to use an intraosseous needle. The intraosseous needle was swiftly placed in the patient's right proximal tibia using an EZ-IO driver (Vidacare, San Antonio, TX; Fig. 1). This 15-gauge device was used for rapid infusion and more than doubled the peripheral access. When the first surgical steps were
A
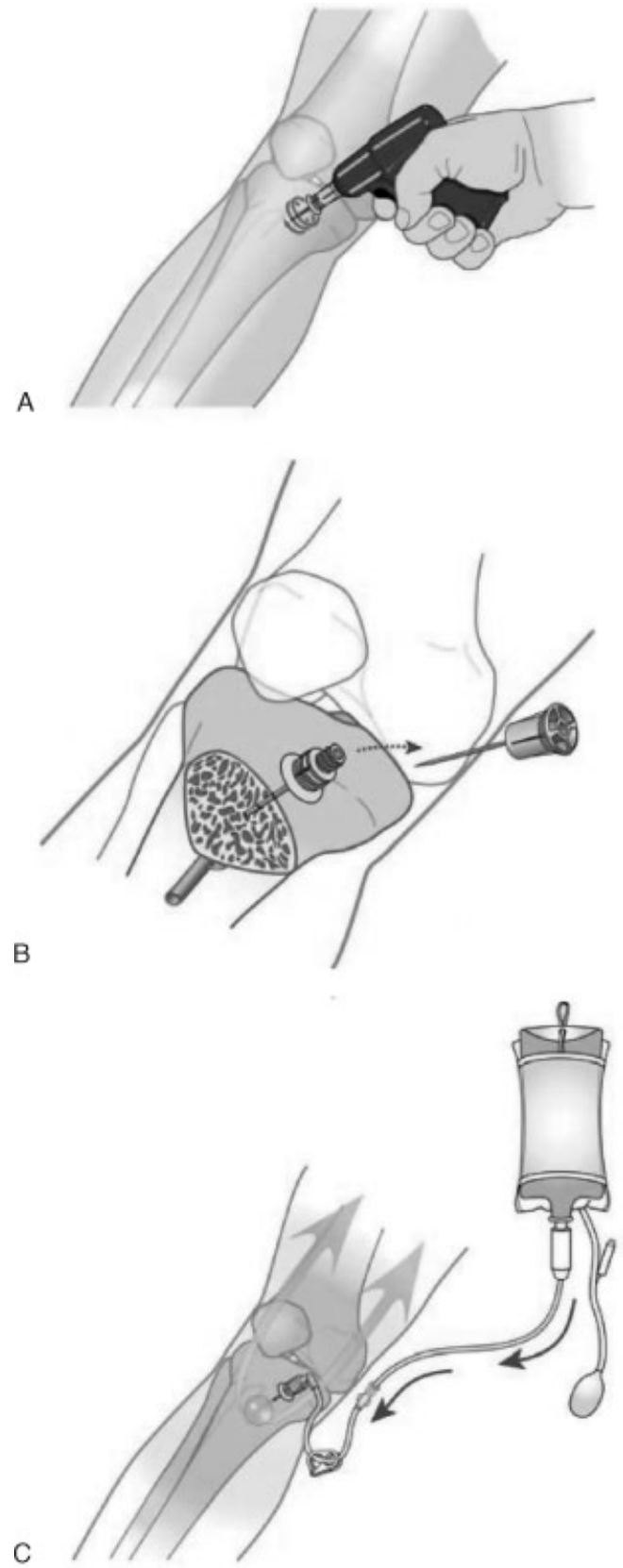

Figure 1 (A-C) Intraosseous needle administration. (Figures reprinted with permission of Vidacare, San Antonio, Texas.)

taken to perform a hysterectomy, the patient suddenly developed a cardiac arrest. Cardiopulmonary resuscitation (CPR) was started and more rapid infusion through the intraosseous needle was administered. As the electrocardiogram demonstrated ventricular fibrillation, the appropriate algorithm of the European Resuscitation Guidelines was followed (http://www.ilcor.org/). ${ }^{8} \mathrm{CPR}$ was performed for $\sim 18$ minutes, with the intraosseous administration of intravenous fluids, epinephrine, and amiodarone. Ventricular defibrillation was performed six times, eventually resulting in restoration of cardiac out- 
put. As the cardiac output returned, the cardiologist was consulted because of suspected failure of the left ventricular function. On cardiac ultrasound, a normal-sized heart was seen without signs of decreased left ventricular function, infarction, or cardiomyopathy. A transesophageal ultrasound probe was placed to assist monitoring of dynamic cardiac output parameters and to assess the adequacy of fluid resuscitation. During the CPR and subsequent otherwise uncomplicated hysterectomy, the abdominal aorta was clamped subdiaphragmatically to maintain coronary and cerebral perfusion during the period of hypovolemic shock and the resuscitation.

When the vital parameters including blood pressure and heart rate were within normal ranges and after completing the hysterectomy, the aortic clamp was removed. After hemorrhage control, intravenous fluid and blood component replacement were provided in collaboration with the anesthesiologist-intensivist. In total, the patient received $20 \mathrm{U}$ packed cells, $10 \mathrm{U}$ fresh frozen plasma, 20 pools of platelets, Novoseven (recombinant activated factor VII; Novo Nordisk, Crawley, West Sussex, UK; $90 \mu \mathrm{g} / \mathrm{kg}$ ) intravenously, $1 \mathrm{~g}$ tranexamic acid intravenously, desmopressin $0.4 \mu \mathrm{g} / \mathrm{kg}\left(\mathrm{DDAVP}{ }^{\mathbb{R}}\right.$; Ferring BV, Hoosddorp, The Netherlands), $3000 \mathrm{U}$ of factor II/VII/IX/X (Co-fact ${ }^{\circledR}$, Sanquin Amsterdam, The Netherlands), 3 L Voluven ${ }^{\circledR}$ (6\% hydroxyethyl starch 130/0.4 in $0.9 \%$ sodium chloride injection; Fresenius Kabi Nederland BV), and $5 \mathrm{~L}$ crystalloid infusion. The estimated blood loss was $12 \mathrm{~L}$. The amount of fluid given through intraosseous access was $75 \%$ of the total infusion volume. All resuscitation medication was administered by the intraosseous route.

After 12 hours, the patient could be detubated on the intensive care unit. Postoperative recovery was uneventful. Overall, she had a full recovery and was discharged home with her son after 8 days.

Postoperative examination of the uterus demonstrated a low uterine segment laceration, which was confirmed histopathologically (Fig. 2). Postpartum hypopituitarism (Sheehan syndrome), a rare complication of massive $\mathrm{PPH}$, was ruled out by testing the pituitary

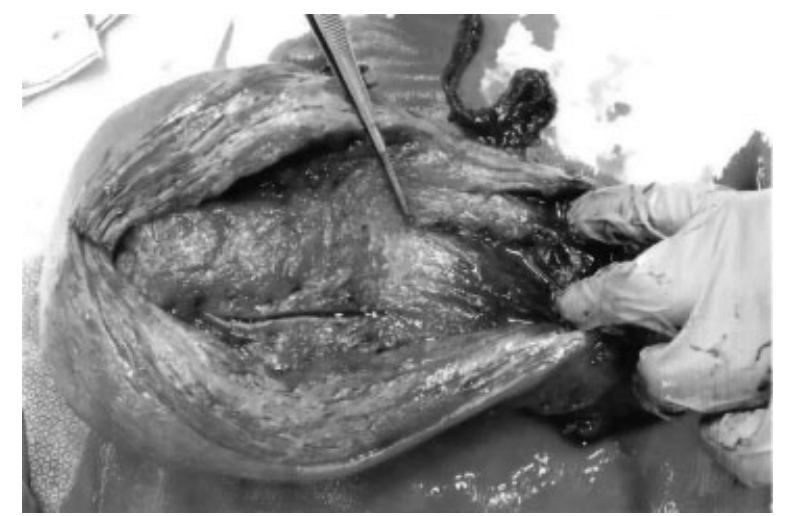

Figure 2 Uterus after hysterectomy with the cervical laceration ending at the tip of the surgical forceps. gland hormones, which demonstrated normal hormone levels 2 months postpartum.

\section{DISCUSSION}

We describe a 42-year-old primigravida with a massive obstetric hemorrhage, ultimately resulting in a postpartum hysterectomy after CPR because of hypovolemic shock as a consequence of uncontrollable hemorrhage. Intraosseous access was a crucial step in the restoration of intravascular volume and the successful resuscitation

Maternal age over 35 is a risk factor for subsequent postpartum hysterectomy. ${ }^{9}$ The Royal College of Obstetricians and Gynaecologists (RCOG) has edited a guideline how to manage $\mathrm{PPH},{ }^{10}$ which is also described in the MOET Course Manual. ${ }^{11}$ Uterine atony is the most frequent course of $\mathrm{PPH}$, and basic management consists of catheterizing the urinary bladder, uterine bimanual massage and compression, and administration of uterotonic drugs. ${ }^{6,10,11}$ Advanced management include hemostatic drugs, uterine balloon tamponade, artery ligation, embolization, uterine compression sutures, and hysterectomy. ${ }^{6,10,11}$ In the United Kingdom, peripartum hysterectomy due to $\mathrm{PPH}$ has a mortality rate of $0.6 \%{ }^{12}$ This case report of massive obstetric hemorrhage is of special interest because in this patient with massive blood loss, the use of the intraosseous infusion contributed significantly to the restoration of intravascular volume and successful CPR and resuscitation, which eventually resulted in stabilization and survival of this patient through the administration of huge amounts of fluids, blood, blood components, and clotting factors through the intraosseous needle.

In the RCOG and Dutch guideline on PPH, ${ }^{10,13}$ intraosseous infusion is not mentioned as a first-line treatment. In recent years, the use of the intraosseous infusion technique to obtain vascular access has been widely advocated; it is also recommended in the most recent Managing Obstetric Emergencies and Trauma (MOET) course (http://www.moetcursus.n1/). This procedure is also endorsed by the American Heart Association and the European Resuscitation Course if intravenous access is difficult or impossible. ${ }^{14,15}$

Although normally considered as an alternative route for vascular access in children, it can also be effective in adults. Intraosseous injection of drugs achieves adequate plasma concentrations in a time comparable with injection through a central intravenous catheter.

The use of intraosseous infusion was first described in the $1930 \mathrm{~s}^{16}$ and is frequently used for pediatric emergencies, ${ }^{17-19}$ where it is sometimes difficult to gain access to the intravascular route. Intraosseous infusion has extensively been used and described in prehospital emergency care, both in children and adults. As it is an effective and safe device for the resuscitation of patients 
in a prehospital setting, the application of intraosseous access should be advocated for in-hospital resuscitation. $^{20}$

Indications for intraosseous vascular access are life-threatening conditions where intravenous or intraosseous access is of great importance such as cardiopulmonary arrest, shock, sepsis, major trauma, or burns. In these situations, use of drugs and major amounts of fluid or blood products are often necessary. Contraindications are rare. Intraosseous needle should not be placed in a long bone with a fracture, or an extremity with a vascular injury or burn. ${ }^{21}$ The success rate of intraosseous insertion varies between $75 \%$ and $100 \%$, with insertion and infusion achieved in most cases within 30 to 120 seconds. ${ }^{17}$ Complications after successful insertion are rare, with an overall incidence of $<1 \%{ }^{21}$ Extravasation of blood, fluid, and drugs is the most common complication and on occasion has led to compartment syndrome. ${ }^{17,22}$ Osteomyelitis can occur but is also rare, with an incidence of $0.6 \%$, mainly due to prolonged infusions. ${ }^{17}$ Other complications with an incidence of $0.7 \%$ are cellulites and skin abscesses. ${ }^{17,23}$ In this case, the successful outcome was achieved by an immediate quick and multidisciplinary team approach by an obstetrician, an anesthesiologist, a surgeon, an intervention radiologist, and a cardiologist and following the MOET protocol for massive obstetric hemorrhage. The hypovolemic shock in this patient eventually led to cardiac arrest, which was successfully managed by intraosseous infusion of fluids and medication through an intraosseous needle. Therefore, we advocate an intraosseous needle in the management of massive PPH when normal intravenous access cannot be obtained quickly; this practice should be included in the protocol handling PPH.

\section{REFERENCES}

1. World Health Organization. WHO Guidelines for the Management of Postpartum Haemorrhage and Retained Placenta. Geneva: World Health Organization; 2009

2. Bais JM, Eskes M, Pel M, Bonsel GJ, Bleker OP. Postpartum haemorrhage in nulliparous women: incidence and risk factors in low and high risk women. A Dutch population-based cohort study on standard ( $>$ or $=500 \mathrm{ml}$ ) and severe $(>$ or $=1000 \mathrm{ml})$ postpartum haemorrhage. Eur J Obstet Gynecol Reprod Biol 2004;115:166-172

3. Jansen AJ, van Rhenen DJ, Steegers EA, Duvekot JJ. Postpartum hemorrhage and transfusion of blood and blood components. Obstet Gynecol Surv 2005;60:663-671

4. Knight M, Callaghan WM, Berg C, et al. Trends in postpartum hemorrhage in high resource countries: a review and recommendations from the International Postpartum Hemorrhage Collaborative Group. BMC Pregnancy Childbirth 2009;9:55

5. Cantwell R, Clutton-Brock T, Cooper G, et al. Saving mothers' lives: reviewing maternal deaths to make mother- hood safer: 2006-2008. The Eighth Report of the Confidential Enquiries into Maternal Deaths in the United Kingdom. BJOG 2011;118(Suppl 1):1-203

6. Chatterjee DJ, Bukunola B, Samuels TL, Induruwage L, Uncles DR. Resuscitation in massive obstetric haemorrhage using an intraosseous needle. Anaesthesia 2011;66:306310

7. Gülmezoglu AM, Forna F, Villar J, Hofmeyr GJ. Prostaglandins for preventing postpartum haemorrhage. Cochrane Database Syst Rev 2007;(3):CD000494

8. Nolan JP, Soar J, Zideman DA, et al; ERC Guidelines Writing Group. European Resuscitation Council Guidelines for Resuscitation 2010 Section 1. Executive summary. Resuscitation 2010;81:1219-1276

9. Knight M, Kurinczuk JJ, Spark P, Brocklehurst P; United Kingdom Obstetric Surveillance System Steering Committee. Cesarean delivery and peripartum hysterectomy. Obstet Gynecol 2008;111:97-105

10. RCOG Green Top Guideline No. 52. Prevention and Management of Postpartum Haemorrhage. London: Royal College of Obstetricians and Gynaecologists; 2009

11. Johanson R, Cox C, Grady K, Howell C. The Moet Course Manual. Managing Obstetric Emergencies and Trauma. 2nd ed. London: RCOG Press; 2007

12. Knight M; UKOSS. Peripartum hysterectomy in the UK: management and outcomes of the associated haemorrhage. BJOG 2007;114:1380-1387

13. Dutch Society of Gynaecology and Obstetrics (NVOG). Guideline Haemorrhagia Postpartum. Utrecht, The Netherlands: NVOG; 2006

14. ECC Committee, Subcommittees and Task Forces of the American Heart Association. 2005 American Heart Association Guidelines for Cardiopulmonary Resuscitation and Emergency Cardiovascular Care. Circulation 2005;112(24, Suppl):IV1-IV203

15. Deakin CD, Nolan JP, Soar J, et al. European Resuscitation Council Guidelines for Resuscitation 2010 Section 4. Adult advanced life support. Resuscitation 2010;81:1305-1352

16. Josefson A. A new method of treatment-intraossal injections. Acta Med Scand 1934;81:550-564

17. Luck RP, Haines C, Mull CC. Intraosseous access. J Emerg Med 2010;39:468-475

18. Sommer A, Weiss M, Deanovic D, Dave M, Neuhaus D. [Intraosseous infusion in the pediatric emergency medical service. Analysis of emergency medical missions 1990-2009]. Anaesthesist 2011;60:125-131

19. Tobias JD, Ross AK. Intraosseous infusions: a review for the anesthesiologist with a focus on pediatric use. Anesth Analg 2010;110:391-401

20. Gerritse BM, Scheffer GJ, Draaisma JM. Prehospital intraosseus access with the bone injection gun by a helicoptertransported emergency medical team. J Trauma 2009;66: 1739-1741

21. Rosetti VA, Thompson BM, Miller J, Mateer JR, Aprahamian C. Intraosseous infusion: an alternative route of pediatric intravascular access. Ann Emerg Med 1985;14: 885-888

22. LaSpada J, Kissoon N, Melker R, Murphy S, Miller G, Peterson R. Extravasation rates and complications of intraosseous needles during gravity and pressure infusion. Crit Care Med 1995;23:2023-2028

23. Fiser DH. Intraosseous infusion. N Engl J Med 1990;322: 1579-1581 